\section{Marcos referenciais da trajetória das políticas de alimentação e nutrição no Brasil}

\section{Milestones of the feeding policies and nutrition in Brazil}

Bertoldo Kruse Grande de Arruda1 Ilma Kruze Grande de Arruda2

\begin{abstract}
The milestones of the concept and implementation of innovative proposals in the field of nutrition appeared in the 30's. The 1933 survey performed by Josué de Castro in Recife focusing the qualify of life of the working classes determined calories' and nutrients' deficiencies and encouraged the development of new researches focusing on the food situation in Pernambuco, Rio de Janeiro and São Paulo, establishing a relationship between food and minimum wage. To assist the government in formulating a National Policy for Food and Nutrition the National Institute for Food and Nutrition (INAN) was founded in 1972 and the I National Program for Food and Nutrition (PRONAN I) was designed comprising 12 sub-programs of different governmental entities. With the innovative concept of malnutrition as a social disease PRONAN I was replaced in 1976 by the PRONAN II. INAN became extinct in 1997 and was replaced by the General Coordination of Feeding and Nutrition Policies, in charge of designing a National Policy for Food and Nutrition in the context of Food and Nutrition Security which, in 2001 created the Food Aid Program. The creation of the National Council of Food Security in 1993 encouraged the approval of the Fome Zero Program (Zero Hunger Program). The Brazilian reality is one traditionally impacted by the lack of systematic procedures.
\end{abstract}

Key words Nutrition programmes and policies, Nutrition programmes, Food security
1 Instituto Materno Infantil Prof. Fernando Figueira - IMIP. Rua dos Coelhos, 300. Recife, PE, Brasil. CEP: 50.070-550. E-mail: bertoldo@imip.org.br

2 Departamento de Nutrição. Universidade Federal de Pernambuco. Recife, PE, Brasil.

\section{Resumo}

Os marcos que influenciaram a concepção e a implementação de propostas inovadoras no campo da nutrição surgiram na década de 30. O inquérito promovido por Josué de Castro no Recife, em 1933, sobre as condições de vida das classes operárias revelou a ocorrência de déficit calórico e de nutrientes e motivou o desenvolvimento de novas pesquisas acerca da situação alimentar em Pernambuco, Rio de Janeiro e São Paulo, estabelecendo as relações entre alimentação e salário mínimo. Para dar assistência ao governo na formulação da Política Nacional de Alimentação e Nutrição foi criado o Instituto Nacional de Alimentação e Nutrição (INAN), em 1972, e elaborado o I Programa Nacional de Alimentação e Nutrição (PRONAN), envolvendo 12 subprogramas das diversas estruturas governamentais e, com olhar inovador da desnutrição como uma doença social foi substituído, em 1976, pelo II PRONAN. A extinção do INAN, em 1997, conduziu ao surgimento da Coordenação Geral da Política de Alimentação e Nutrição, responsável pela elaboração da Política Nacional de Alimentação e Nutrição no contexto da Segurança Alimentar e Nutricional, criando em 2001 o Programa Bolsa Alimentação. A criação do Conselho Nacional de Segurança Alimentar, em 1993, fomentou a aprovação do Programa Fome Zero. Identifica-se que na realidade brasileira, a avaliação das políticas públicas é um campo tradicionalmente marcado pela carência de procedimentos sistemáticos.

Palavras-chave Programas e políticas de Nutrição e Alimentação,Programas de Nutrição, Segurança alimentar e nutricional 
O mundo globalizado vem se caracterizando por situações contrastantes, pois à medida que se multiplicam as oportunidades derivadas principalmente dos progressos da tecnologia se observam acentuadas tendências excludentes. Todavia, identifica-se um fato positivo - a redescoberta da importância do capital humano e uma maior preocupação com o desenvolvimento social. Na sociedade brasileira, visualiza-se um anseio generalizado de tornar cada vez mais real a expectativa de mudanças, evidenciase a aspiração ético-política de sair da rotina do conformismo e buscar uma nova visão de futuro pelo estímulo às capacidades de antecipação e de perspectiva. Isto conduz à necessidade de evitar respostas lentas, ineficazes e pouco inovadoras aos reclamos de transformações, particularmente no atinente às situações de insegurança e vulnerabilidade alimentares, as quais afetam não somente as condições nutricionais e de saúde da população, mas também preocupam por sua influência na ampliação da trama complexa das tensões sociais e sua crescente interdependência em relação a questões como a estabilidade política e a prevenção de conflitos e da violência. Por isso adquire excepcional relevo a XXXII Reunião do Comitê Permanente de Nutrição da Organização das Nações Unidas (ONU), que se realizou em março de 2005 em Brasília, objetivando consensualizar o pensamento e ações relacionadas à segurança alimentar e nutricional, ana-lisar tendências e reavaliar papéis de setores governamentais e de instituições, visando conseguir atos concretos e coerentes de empenho político.

A compreensão da trajetória das políticas de alimentação e nutrição no Brasil requer, conforme explicita Prado (1993: 23): 1

[...] entender a conjuntura econômica, política e social

do país, no momento histórico em que foram implantadas.

Diante da diversidade, amplitude e complexidade dos cenários que compõem a historiografia da alimentação e nutrição em nosso país, objeto de muitas contribuições, focalizamos os marcos mais expressivos que, a nosso ver, influenciaram e balizaram a concepção e a implementação de algumas propostas inovadoras, a partir da década de 30. Nessa época, adquire ressonância a realização no Recife, em 1933, do inquérito promovido por Josué de Castro, com o patrocínio do Departamento de Saúde Pública de Pernambuco, sobre "as condições de vida das classes operárias", revelando a ocorrência de déficit calórico e de nutrientes, estudo que foi aprofundado em 1935 com o apoio do Departamento Nacional de Saúde Pública, e motivou o desenvolvimento de novas pesquisas, a partir de
1936, acerca da situação alimentar em Pernambuco, entre as quais destaca-se a conduzida por Lima $e t$ $a l .{ }^{2}$ em populações faveladas.

A alusão a essa etapa da trajetória das políticas de alimentação e nutrição em nosso país leva a ressaltar a figura de um pensador original e polêmico, o professor Josué de Castro, que associou, harmoniosamente, a sua capacidade de argumentar à segurança científica, revelando-se detentor de um horizonte intelectual que não se enquadrava nos estreitos limites de uma disciplina acadêmica. O seu propósito, ao apreciar regionalmente o problema da fome no Brasil, era incorporar características fundamentais do humanismo aos projetos de desenvolvimento, era colocar o conhecimento científico a serviço da ação política e da defesa das classes menos favorecidas. Este, no entender de Arruda, 3 é o cerne da mensagem estimuladora e perturbadora de Geografia da Fome, que exibiu um novo quadro contextual e conceitual, com base nas especificidades regionais, integrando natureza, cultura e condicionamentos sócio-políticos dentro de um mesmo campo de interações, abriu novos caminhos para aqueles que buscam a correção de desequilíbrios regionais e a superação do subdesenvolvimento e, apreciando regionalmente o problema da fome, contribuiu para compor um mapeamento caracterizador da sua universalidade. Os delineamentos conceituais e propositivos de Josué de Castro ainda constituem orientação indispensável para se pensar criticamente a realidade alimentar e nutricional brasileira.

Inúmeras contribuições surgiram não só em Pernambuco e também no Rio de Janeiro e em São Paulo, que despertaram, na percepção de Castro (1977: 112):4

[...] a consciência nacional para o angustiante problema da nutrição e, mais do que isto, suas implicações para o processo produtivo.

Daí a afirmação dessa autora de que um dos resultados desses estudos, ao estabelecer as relações entre alimentação e salário, intensificou na imprensa a campanha pelo salário mínimo. Assim, em abril de 1938, com o Decreto-Lei $\mathrm{n}^{\circ} 399$ foi aprovado o salário mínimo, cujos valores foram fixados em $1^{\circ}$ de março de 1940 pelo Decreto-Lei n ${ }^{\circ} 2162$, iniciativa que, à época, iria beneficiar $58 \%$ da população assalariada. ${ }^{4}$ Contemporânea a esse fato marcante foi a criação do Serviço de Alimentação da Previdência Social (SAPS), pelo Decreto-Lei $n^{\circ} 2478$, de agosto de 1940, com finalidades mais amplas: propiciar instalações e as condições para a alimentação adequada dos trabalhadores, fornecer os alimentos a preços acessíveis, capacitar pessoal para as atividades de nutrição e promover a educação alimentar, 
que não se restringia aos operários e alcançava a família dos mesmos, mediante visitas realizadas pelas visitadoras de alimentação. ${ }^{5}$ O SAPS foi objeto de reorganização em 1941 e em 1942, sendo extinto em dezembro de 1967. Dois outros organismos nasceram na década de 40: em 1944, o Instituto Técnico de Alimentação (ITA), subordinado à Coordenação de Mobilização Econômica, que em 1946 é transformado em Instituto Nacional de Nutrição da então Universidade do Brasil; em fevereiro de 1945, pelo Decreto-Lei n. 7328, é criada junto ao Conselho Federal de Comércio Exterior a Comissão Nacional de Alimentação (CNA), transferida em 1949 para o Ministério da Educação e Saúde, investida em abril de 1951 nas funções de Comitê Nacional da Food and Agriculture Organization (FAO) e, em agosto de 1951, incumbida de assistir o governo na formulação da política nacional de alimentação, sendo extinta em 1972, quando da criação do Instituto Nacional de Alimentação e Nutrição (INAN). Em 1956, Nelson Chaves fundou o Instituto de Nutrição, atualmente Departamento de Nutrição da Universidade Federal de Pernambuco, e advogava o fortalecimento do tripé nutrição/saúde/educação, objetivando compreender a realidade social nordestina e encontrar soluções para a reconstrução dessa realidade.

No início dos anos 70 ampliou-se a discussão sobre a influência da nutrição como objetivo explícito e deliberado do desenvolvimento, frente à constatação de que a solução dos problemas não pode ser obtida pelo simples acionamento do instrumental de ações próprias de um único setor, a saúde ou a agricultura. Representou importante contributo para a tomada de decisão governamental uma das recomendações da III Reunião Especial dos Ministros de Saúde das Américas, realizada em Santiago do Chile, em 1972, assinalando ser necessário efetuar mudanças na condução das políticas sociais. 6 Gerou-se, então, o clima favorável para a criação do Instituto Nacional de Alimentação e Nutrição (INAN), efetivada pela Lei $\mathrm{n}^{\circ} .5829$ de novembro de 1972, como autarquia vinculada ao Ministério da Saúde, que assumiu as atribuições da extinta Comissão Nacional de Alimentação com a finalidade de: a) assistir o Governo na formulação da política nacional de alimentação e nutrição; b) elaborar e propor ao Presidente da República o Programa Nacional de Alimentação e Nutrição (PRONAN), promover sua execução, supervisionar e fiscalizar sua implementação, avaliar periodicamente os respectivos resultados e, se necessário, propor revisão; e c) funcionar como órgão central das atividades de alimentação e nutrição.7,8

Em março de 1973, pelo Decreto-Lei no 72.034, foi instituído o I PRONAN, constando de um elenco de 12 subprogramas, integrantes das diversas estruturas governamentais, com vigência até 1974, cujo desempenho transcorreu com dificuldades e foi interrompido em face das transgressões normativas e operativas constatadas pelas auditorias procedidas no Instituto. ${ }^{9}$ Como decorrência do início de uma nova gestão administrativa, em janeiro de 1975 foi celebrado convênio entre o INAN e o Instituto de Pesquisa Econômica e Aplicada (IPEA), ${ }^{10}$ formalizando-se a constituição de equipe técnica para realizar estudos no campo da alimentação e nutrição e definir o II PRONAN, tendo presentes os delineamentos estabelecidos no II Plano Nacional de Desenvolvimento (II PND, 1975/1979), que reformulou a visão e a atuação governamental no campo social, com base em três princípios: a) o da igualdade hierárquica entre o desenvolvimento social e o econômico e da simultaneidade dos dois processos; b) o do tratamento privilegiado para os grupos de baixa renda, considerados objeto principal da política social; e c) o da responsabilidade compartida entre a área social e a área econômica na solução dos problemas sociais.

Em fins de 1975, o texto básico elaborado pelo grupo INAN E IPEA, contendo estratégias e áreas de ação até então pouco consideradas, foi debatido em Seminário com participantes das áreas acadêmica, governamental e empresarial, sendo de justiça destacar seis lideranças: Nelson Chaves, Mário Magalhães da Silveira, Eduardo Kertézs, Fernando Figueira, Yaro Ribeiro Gandra e Alberto Carvalho da Silva.11 Posteriormente, em fevereiro de 1976, ocorreu a aprovação pelo Decreto n. 77.116 do II Programa Nacional de Alimentação e Nutrição (II PRONAN), com vigência correspondente ao II PND, que traduziu um novo comportamento políticoadministrativo e revelou-se inovador ao conceber a desnutrição como uma doença social.12 A formulação do II PRONAN, objetivando a atuação integrada para o máximo proveito de todas as possibilidades de intervenção, a fim de conseguir um impacto significativo, partiu dos seguintes pressupostos: a) a população-alvo deve ser escolhida com base na vulnerabilidade social, considerando como variável determinante a renda e como limite a renda familiar de até dois salários mínimos; b) a prioridade de faixa etária deve considerar a vulnerabilidade biológica, para atendimento ao período de vida do ser humano em que ele é, por condições fisiológicas, mais vulnerável às agressões das deficiências nutricionais; c) a seleção do suplemento alimentar deve dar preferência aos alimentos tradicionais, levando em consideração que a dieta de quase todas as pes- 
soas gira em torno de um grupo reduzido de alimentos, dos quais deriva a maior parte das calorias e proteínas; d) a opção por alimentos básicos fundamenta-se também no seu custo comparativo mais baixo, somado à necessidade de estimular os pequenos e médios produtores rurais, que constituem o núcleo de produção desses alimentos, canalizando, dessa maneira, o volume de compra dos programas de suplementação alimentar para assegurar uma demanda aos pequenos produtores e reduzir, ao mesmo tempo, os canais de comercialização do que produzem. A área prioritária de atuação foi o Nordeste, em virtude da alta prevalência dos problemas nutricionais e das perspectivas de incremento da potencialidade produtora dos pequenos agricultores, os quais aumentariam sua renda e o consumo de alimentos, reorientando a sua produção para o mercado, em vez de produzirem predominantemente para seu próprio consumo. ${ }^{9}$

Essa orientação encontrava respaldo no II PND, que reconhecia não ser o desenvolvimento social uma conseqüência direta e automática do crescimento econômico e, portanto, o Estado devia interferir para assegurar aos contingentes populacionais mais carentes, o acesso aos bens e serviços essenciais. As diretrizes do II PRONAN13 conduziram à consolidação da seguinte estrutura programática:

Programas sob a gestão direta do INAN

Programa de Nutrição em Saúde (PNS), que distribuía alimentos in natura, cobrindo $45 \%$ das necessidades nutricionais diárias de crianças, gestantes e nutrizes, integrando o elenco de ações básicas de saúde;

Programa de Alimentos Básicos em Áreas de Baixa Renda (PROAB), que abastecia os pequenos varejistas de áreas carentes, com alimentos básicos e preços reduzidos;

Programa de Racionalização da Produção de Alimentos Básicos (PROCAB), que adquiria os alimentos básicos diretamente do produtor, por intermédio da Companhia Brasileira de Alimentos (COBAL) do Ministério da Agricultura, para programas do PRONAN;

Programa Nacional de Incentivo ao Aleitamento Materno;

Programa de Combate às Carências Específicas, incluindo a prevenção e tratamento do bócio endêmico, da hipovitaminose A, da anemia ferropriva e da cárie dental;

Sistema de Vigilância Alimentar e Nutricional, cujo modelo foi testado em Pernambuco, mas não chegou a ser implantado.

Programas dependentes de outros órgãos:

Programa Nacional de Alimentação Esco- lar (PNAE), que funcionava desde 1954, sob a coordenação da Campanha Nacional de Alimentação Escolar (CNAE) e, posteriormente, da Fundação de Assistência ao Estudante (FAE), do Ministério da Educação e Cultura, provendo merenda para escolares de 7 a 14 anos de idade;

Programa de Complementação Alimentar (PCA), sob a coordenação da Legião Brasileira de Assistência (LBA), do Ministério da Previdência, que atendia com alimentos formulados a sua rede assistencial;

Programa de Alimentação do Trabalhador (PAT), criado em 1977, sob a coordenação do Ministério do Trabalho, que mediante incentivo fiscal, possibilitava às empresas fornecer refeições aos trabalhadores.

Atividades de complementação e apoio:

Estudos e pesquisas, componente ampliado pelo Projeto de Nutrição Brasil/Bird (PNBB), com base no acordo celebrado entre o Governo Brasileiro e o Banco Mundial;

Formação de recursos humanos, com ênfase no apoio a cursos de nutrição, que visou à reorientação da formação e/ou capacitação profissional, em nível de graduação e pós-graduação.

Esse conjunto de atividades, que implicava promover articulações com organismos subordinados a outros Ministérios, constituiu uma estratégia que apresentou dificuldades operacionais, das quais se teve consciência quando da formulação do II PRONAN, porque não se buscava o mais fácil de operacionalizar e sim o que efetivamente devia ser realizado para alcançar o desiderato pretendido de justiça social. Vale salientar os principais desafios: a) a política de fortalecimento do pequeno produtor, o grande responsável pelo abastecimento de alimentos básicos no Brasil. Reconhecendo na suplementação alimentar um estímulo à produção de alimentos básicos e expansão do mercado consumidor nas áreas menos favorecidas, objetivou-se, por meio da garantia de compra da produção desses pequenos agricultores, desencadear uma transformação no campo de consequiências benéficas. Para tanto, fazia-se necessário rever as diretrizes relativas a subsídio, crédito, financiamento, assistência técnica, pesquisa, preços mínimos, seguros, etc, no sentido de fortalecê-los e assegurar impacto social mais significativo; b) o desenvolvimento harmônico do II PRONAN foi comprometido pelo fato de os recursos orçamentários ficarem vinculados aos Ministérios responsáveis pela execução de seus programas e projetos, ocorrendo, às vezes, reduções que prejudicavam o desempenho, porque cada Ministério tinha autonomia para definir sua estrutura 
programática e atribuir valores orçamentários; c) em face de o INAN ter sido uma agência setorial vinculada ao Ministério da Saúde, a incumbência de coordenação do PRONAN oferecia obstáculos pela impossibilidade de interferência sobre determinados problemas gerados pelo descompasso entre as políticas estabelecidas e suas efetivas aplicações, faltando-lhe mecanismos adequados para influir em outros setores comprometidos com a execução do PRONAN.

Aproximando-se o término da vigência do II PRONAN, com base na experiência dos diversos órgãos da área social e econômica responsáveis pela sua execução, foi elaborada a proposta do III PRONAN, com vistas a dar à problemática alimentar e nutricional uma atenção mais consentânea de sua real dimensão, selecionando modelos de intervenção que foram exaustivamente testados durante o funcionamento da programação e a articulação interinstitucional que a viabilizava técnica, operacional e financeiramente8. Após apreciação pelo Conselho Deliberativo do INAN, em maio de 1981, o anteprojeto do III PRONAN foi encaminhado à instância superior para aprovação, a qual não ocorreu e os programas do II PRONAN continuaram a ser executados, com perda acentuada da sua importância. Em 1985, o Programa de Nutrição em Saúde (PNS) do INAN passa a se denominar Programa de Suplementação Alimentar (PSA) e em 1986, pelo Decreto-lei n. 93.120, é regulamentada a implantação do Programa Nacional do Leite para Crianças Carentes (PNLCC), ligado à Presidência da República, os quais praticamente foram desativados em 1992.14 A progressiva debilitação dos programas a cargo do INAN conduziu à sua extinção em julho de 1997, embora algumas tentativas para resgatar a relevância institucional da área de alimentação e nutrição tenham sido feitas, uma delas a sua transformação em Secretaria, incorporada na estrutura do Ministério da Saúde. Nessa transição, as atividades ficaram a cargo de uma Área Técnica de Alimentação e Nutrição (ATAN), no âmbito da Secretaria de Políticas de Saúde, e com a extinção dessa Secretaria, a ATAN ficou no Departamento de Atenção Básica da Secretaria de Assistência à Saúde, passando a denominar-se Coordenação Geral da Política de Alimentação e Nutrição (CGPAN).

Destacam-se duas proposições nesse período: uma, a elaboração da Política Nacional de Alimentação e Nutrição, iniciada em 1997, a cargo de técnicos dos setores de saúde, educação e agricultura e consultores, com texto final apresentado e discutido em seminário patrocinado pela Organização Pan-Americana da Saúde (OPAS), com a participação de representações de entidades acadêmicas e de classe, e também de organizações comunitárias. Nesse texto, aprovado pela Portaria n. 710, de junho de 1999, do Ministério da Saúde, está explícito que essa Política integra a Política Nacional de Saúde e inserese, ao mesmo tempo, no contexto da Seguran-ça Alimentar e Nutricional. Os componentes dessa Política revelam o intuito de garantia do direito humano à alimentação e nutrição, bem como denotam que as práticas para alcançá-lo extrapolam os limites do setor saúde e implicam ampla articulação com outros setores governamentais e segmentos da sociedade e do setor produtivo. ${ }^{8}$ A outra proposição, em agosto de 2001, a criação do Bolsa Alimentação, substituiu as ações promovidas sob o rótulo de Incentivo ao Combate às Carências Nutricionais (ICCN). Essa proposição, que foi gra-dualmente desativada, conferiu coerência, amplitude e consistência à programação, mediante o repasse de recurso financeiro às famílias cadastradas, a fim de adquirirem os alimentos e prevendo a condicionalidade do benefício ao cumprimento, pelo beneficiado, de um conjunto de ações básicas de saúde, a agenda de compromissos. Na sua concepção e operacionalização significou um avanço, porque focalizava a atenção nos mais pobres, requeria a co-responsabilidade das famílias e a participação dos conselhos de saúde, estimulando a construção e o fortalecimento da cidadania, concorrendo, assim, para reforço e qualificação da rede básica de saúde. Outrossim, o repasse financeiro direto às famílias cadastradas flexibilizava os tipos de alimentos a serem adquiridos e reduzia os gastos operacionais em nível de município, verificando-se que:

[...] as famílias efetuaram mais gastos com alimentos e

adotaram uma dieta mais diversificada, e [...] as crianças

beneficiárias apresentaram uma melhor recuperaçãonu-

tricional e maior crescimento.(Carvalho et al. 1995: 98,

101).9

$\mathrm{Na}$ seqüência de momentos relevantes em termos de definição de conceitos, procedimentos e deliberações nesse campo, cabe rememorar: a) a VIII Conferência Nacional de Saúde, em 1986 em Brasília, cujas recomendações conduziram à aprovação da Lei Orgânica da Saúde (Lei n. 8080/90), criando o Sistema Único de Saúde e contemplando a estruturação de Comissões Permanentes, entre elas a Comissão Intersetorial de Alimentação e Nutrição do Conselho Nacional de Saúde;6 b) a I Conferência Nacional de Alimentação e Nutrição em Brasília, como desdobramento da VIII Conferência Nacional de Saúde, que propôs a criação de um Conselho Nacional de Alimentação e Nutrição (CNAN) e de um Sistema de Segurança Alimentar e Nutricional 
(SSAN), ambos ligados ao Ministério do Planejamento. Nessas proposições ficou clara a ampliação do conceito de segurança alimentar com a incorporação de conteúdos nutricionais; e c) a Ação da Cidadania contra a Fome, à Miséria e pela Vida, onde desempenhou papel de relevo o sociólogo Herbert de Souza. Resultaram desse movimento: a) a confecção do Mapa da Fome;16,17 b) a elaboração do Plano de Combate à Fome e à Miséria, 18 e a criação do Conselho Nacional de Segurança Alimentar em maio de 1993; e c) a realização em Brasília, em julho de 1994, da I Conferência Nacional de Segurança Alimentar, que induziu um processo de mobilização nacional em torno da questão alimentar e da dimensão do problema da fome no país. Essa Conferência consolidou três aspectos básicos: a) o processo de desenvolvimento econômico e social do país necessita garantir, obrigatoriamente, a segurança alimentar para todos; b) a proposta de segurança alimentar exige uma arti-culação entre a sociedade civil e o governo; e c) a participação conjunta não implica diluição dos papéis específicos que cabem a cada um dos participantes.

Em janeiro de 2003, iniciando-se um novo período governamental, as ações relacionadas à segurança alimentar são colocadas no centro de uma política de desenvolvimento, objetivando garantir quantidade, qualidade e regularidade no acesso à alimentação para toda a população brasileira. Essa iniciativa tomou por base os estudos do Instituto da Cidadania, 19 sendo aprovado o Programa Fome Zero, em cuja estruturação distinguimos três dimensões importantes: a) a teóricoconceitual, reconhecendo que a formulação de uma política de segurança alimentar irá constituir o marco de referência idealmente desejável para o Programa, a fim de configurar uma abordagem holística; b) a político-operativa, consubstanciada na decisão de implementá-lo e gradativamente promover ajustes, e na criação do Ministério Extraordinário da Segurança Alimentar e Combate à Fome; e c) a consultiva, recriando o Conselho Nacional de Segurança Alimentar (CONSEA), como instância de acompanhamento e catalisadora de articulação inter-setorial, retomando experiência iniciada em 1993 e interrompida em 1995.

Na percepção de Frei Betto (2004: 23).20

[...] o Fome Zero não é assistencialista, nem se resume a

ações emergenciais. Trata-se de uma política de inserção

social, para a qual, mais importante do que distribuir

alimentos, é gerar renda, trabalho, resgatar a auto-estima

e a cidadania.

Sem dúvida, representou um fato promissor essa tomada de posição frente às dimensões e determinantes da fome, porque a construção de uma consciência política constitui o pré-requisito para um tratamento adequado do problema. Entretanto, não basta a decisão política, pois essa vontade arrefecerá, a menos que seja seguida por ações de impacto, que têm como pré-condições a adequação das estruturas existentes, a concessão oportuna de recursos financeiros, o planejamento e execução harmônica dos programas, e o monitoramento, avaliação e divulgação dos resultados. No caso do Fome Zero, pela sua abrangência em cerca de 60 ações, no entender de Belik, ${ }^{21}$ um de seus idealizadores, "é um guardachuva de iniciativas" e "é um patrimônio que legitima a ação do governo e sacode a inércia da sociedade".22 As ações estão enfeixadas em três eixos de atuação: a) políticas estruturais, voltadas para o combate às causas da fome e da pobreza: políticas emergenciais, para enfrentar situações de insegurança alimentar de segmentos específicos da sociedade com insuficiência de renda; e políticas locais, administradas pelos Estados e Municípios, em conjunto com a sociedade civil organizada.

As dificuldades surgidas impediram ao Ministério Extraordinário da Segurança Alimentar e Combate à Fome, o satisfatório desempenho do seu papel de articulador, pois se apresentavam grandes desafios: a) superar a visão de que segurança alimentar e nutricional é a mesma coisa que combate à fome; b) superar a visão de que a Política de Segurança Alimentar e Nutricional é uma política setorial, fazendo-se necessário agregar experiências e ter flexibilidade para compreender as especificidades regionais; c) superar a visão paternalista/assistencialista que permeia a sociedade brasileira e as ações governamentais, cabendo ativar a participação comunitária, para que os usuários utilizem os serviços, ajudem a promovê-los e sugiram mudanças; d) superar a incompreensão da importância do desenvolvimento de ações de segurança alimentar e nutricional em todos os níveis, e de que a sociedade organizada em Estado tem obrigações em relação à garantia do direito humano à alimentação e nutrição para todos, mas que todas as pessoas também têm responsabilidades.

Os percalços operativos na condução do Fome Zero, atualmente já relacionando 81 ações, induziram a um reposicionamento do Governo em relação às diretrizes fixadas, cujo desdobramento foi a unificação dos quatro programas de transferência de renda - Bolsa-Escola, Bolsa-Alimentação, AuxílioGás e Cartão-Alimentação, instituindo pela Lei $\mathrm{n}^{\circ}$ 10.836, de janeiro de 2004,23 o Programa Bolsa Família, que manteve as condicionalidades previstas para os Programas Bolsa-Escola e Bolsa-Alimentação, ficando no âmbito do Ministério do Desenvolvimento Social e Combate à Fome, que sucedeu 
ao aludido Ministério Extraordinário. Trata-se, em essência, de uma transformação com o objetivo de aumentar a eficiência e o impacto social dessa ação, corrigindo e aperfeiçoando o que se fazia necessário nos programas existentes, visando conferir maior racionalidade à concessão dos benefícios e condições para melhor controle. O elenco de argumentos convergentes que balizou essa reformulação programática demonstrou o surgimento de uma alternativa convincente, mas que suscitou, por parte de analistas, notadamente da área econômica, algumas ponderações, em especial acerca da possibilidade dos atendidos pelo Bolsa-Família não romperem o ciclo de dependência do Estado, e ser essencial o desenvolvimento de um modelo de avaliação capaz de identificar as origens dos desperdícios e de manter um diálogo com os demais programas governamentais.

Aliás, a avaliação das políticas públicas, em nosso país, ainda é um campo tradicionalmente marcado pela carência de procedimentos sistemáticos, que possibilitem concluir sobre o impacto e a implementação. Identificam-se esforços numerosos e bem intencionados, admitem Lavinas e Garcia,24 mas pouco há a dizer sobre sua eficácia. Entre os fatores impeditivos da implementação efetiva, Valente (2004: 104)25 refere:

[...] inadequação de mecanismos estaduais e municipais de gestão que possam garantir que a população mais carente seja efetivamente atendida pelos programas em andamento.

Outra necessidade é o fortalecimento dos conselhos de controle social, fazendo com que os mesmos deixem de ser interpretados, freqüentemente, como instrumentos de validação das ações governamentais. No entanto, diante da exigência generalizada de participação sempre mais ampla, convém proceder cautelosamente para que os conselhos locais não se transformem em cenários de encenação de democracia participativa, bem como regularmente se capacitarem para facilitar o encurtamento da distância entre as necessidades sentidas e as soluções esperadas, convencidos do que preceitua Touraine "É tão perigoso se limitar às críticas quanto fazer propostas estranhas à realidade.” (2002: 8-9).26
Cabe o registro de um outro acontecimento auspicioso, a realização da II Conferência Nacional de Segurança Alimentar e Nutricional em Olinda, Pernambuco, em março de 2004, decorridos 10 anos da I Conferência.27 Os ensinamentos que proporcionou constituem parâmetros para uma participação efetiva na direção correta, na aplicação diligente de bons preceitos e técnicas, com vistas ao atendimento preventivo/corretivo das várias faces da insegurança alimentar. Convém ressaltar que entre as conclusões e recomendações dessa II Conferência é colocada:

[...] a segurança alimentar e nutricional como objetivo

estratégico e permanente associado à soberania

alimentar"; [...] respeitar a equidade de gênero e étnica,

reconhecendo a diversidade e valorizando as culturas

alimentares. (II Conferencia Nacional de Segurança

Alimentar ...; 2004: 5).27

Deve-se buscar a transversalidade das ações por intermédio de planos articulados intersetorialmente e com participação social.27

As revisitações contidas neste breve percurso rememorativo levam a reflexionar acerca da pertinência da conclusão a que chegou Pena (2004: 107).28

[...] o desafio do desenvolvimento social contemporâneo no Brasil permanece o de cumprir com três objetivos principais: não empobrecer os que não são pobres; criar canais de ascenção para os grupos sociais mais desfavorecidos, intervindo nos processos que criam sua vulnerabilidade; promover o direito à vida dos mais pobres até que possam deixar de sê-lo.

Na perspectiva desta história o que importa é não prevalecerem as declarações de boas intenções, porém aumentar a margem para otimismo quanto às alternativas concretas de intervenção nas prioridades fixadas e diminuir o distanciamento entre as intenções e as ações efetivas a tornar realidade para todos a segurança alimentar. Afinal, sentenciou Josué de Castro (1984: 34):29

[...] a fome traduz sempre um sentimento de culpa, uma prova evidente de que as organizações sociais vigentes se encontram incapazes de satisfazer a mais fundamental das necessidades humanas - a necessidade de alimentos. 


\section{Referências}

1. Prado MS. A trajetória da política de alimentação no Brasil: de 1889 a 1945. Rev Baiana Saúde Publica. 1993; 20: 23 31.

2. Lima JF, Melo FB de, Costa F. Algumas medidas para a melhoria do abastecimento regional. In: Lima JF, Melo FB, Costa F, Bancosvsky J, coordenadores. Aspectos do problema alimentar no Nordeste. Recife: Imprensa Universitária; 1962. p. 48-73.

3. Arruda BKG. Geografia da fome: da lógica regional à universalidade. Cad Saúde Publica. 1997; 13: 545-9.

4. Castro AM. Nutrição e desenvolvimento. Análise de uma política [tese livre-docência]. Rio de Janeiro: Instituto de Nutrição da Universidade Federal do Rio de Janeiro; 1977.

5. L'Abbate S. As políticas de alimentação e nutrição no Brasil. I período de 1940-1964. Rev. Nutr PUCAMP. [Campinas], 1988; 1: 87-138.

6. OPS (Organización Panamericana de la Salud), OMS (Organização Mundial de la Salud). Plan Decenal para las Américas: informe final de la III Reunión Especial de Ministros de Salud de las Américas.; 1972 out 2-9; Santiago, Chile. Santiago; 1973. (Documento Oficial, n. 118).

7. Brasil. Senado Federal. Criação do Instituto Nacional de Alimentação e Nutrição - INAN e dá outras providências. Disponível em: www6.senado.gov.br/sicon/listareferenciaaction? codigobare $=2 \&$ codigodcum [2007 ago 8].

8. Batista Filho M, Barbosa NP. Alimentação e nutrição no Brasil: 1974-1984. Brasília, DF: Ministério da Saúde; 1985.

9. Carvalho da Silva A. De Vargas a Itamar: políticas e programas de alimentação e nutrição. Estud Av. 1995; 9: 87-107.

10. Castro CM, Coimbra M, organizadores. O problema alimentar no Brasil. São Paulo: Editora UNICAMP; 1985.

11. Brasil. Presidência da República. II Plano Nacional de Desenvolvimento: 1975-1979. Disponível em: www.inep.gov.br/pesquisa/bbe-online/det.asp? $\operatorname{cod}=102878$ type $=\mathrm{m}[2007$ jul 4].

12. Pessanha LDR. A experiência brasileira em políticas públicas para a garantia do direito ao alimento. Rio de Janeiro: Departamento de Ensino e Pesquisa da Escola Nacional de Ciências Estatísticas; 2002.

13. Arruda BKG. O Programa Nacional de Alimentação e Nutrição (PRONAN) In: Chaves, N. Nutrição básica e aplicada. 2. ed. Rio de Janeiro: Guanabara Koogan; 1985

14. Conh A. Políticas sociais e pobreza no Brasil. Plan Pol Públicas. 1995; 12: 1-18.

15. Brasil. Ministério da Saúde. Departamento de Atenção Básica. Coordenação Geral da Política de Alimentação e Nutrição. Avaliação do Programa Bolsa-Família: segunda fase. Brasília, DF; 2005. (Série C. Projetos, Programas e Relatórios).
16. IPEA (Instituto de Pesquisa Econômica e Aplicada). O mapa da fome: subsídios à formulação de uma política de segurança alimentar. Brasília, DF; 1993. (Documento de Política, n. 14).

17. IPEA (Instituto de Pesquisa Econômica e Aplicada). O mapa da fome II: informações sobre a indigência por municípios da federação. Brasília, DF; 1993. (Documento de Política, n. 15).

18. IPEA (Instituto de Pesquisa Econômica e Aplicada). Plano de combate à fome e à miséria: princípios, prioridades e mapa das ações de Governo. Brasília, DF; 1993.

19. Universidade de Campinas. Instituto Cidadania. Projeto Fome Zero: uma política de segurança alimentar para o Brasil. Campinas; 2000.

20. Betto, Frei. A fome como questão política. In: Betto Frei, organizador. Fome Zero: textos fundamentais. Rio de Janeiro: Garamond; 2004.

21. Belik W. A medida da pobreza. Folha de São Paulo. 2003 abr 10; Sec Opinião: A3.

22. Belik W. Uma ponte entre dois mundos. Folha de São Paulo. 2003 set; Sec Opinião: A3.

23. Brasil. Controladoria Geral da União. [unificação de programas]. Disponível em: www81.dataprev.gov.br/sislex. [2004 jun 18].

24. Lavinas L, Garcia EH. Programas sociais de combate à fome: o legado dos anos de estabilização econômica. Rio de Janeiro: Editora Universidade Federal do Rio; 2004.

25. Valente FLS. O direito à alimentação. In: Lima Jr JB, Zetterström L, organizadores. Extrema pobreza no Brasil: a situação do direito à alimentação e moradia adequada. São Paulo: Loyola; 2004.

26. Touraine A. A política contra a cegueira. Folha de São Paulo. 2002. jan 27; Cad Mais: 8-9.

27. II Conferência Nacional de Segurança Alimentar e Nutricional em Olinda: relatório final; 2004 17-20 mar.; Olinda, Pernambuco. Olinda; Ministério da Saúde; 2004.

28. Pena MVJ. Os desafios das políticas de combate à pobreza. In: Velloso JR, Albuquerque RC, coordenadores. A nova geografia da fome e da pobreza. Rio de Janeiro: José Olympio, 2004. p. 101-8.

29. Castro J. Geografia da fome (o dilema brasileiro: pão ou aço). 10. ed. revista. Rio de Janeiro: Antares; 1984.

Recebido em janeiro de 2007

Versão final em 2 julho de 2007

Aprovado em 26 de julho de 2007 